\title{
Optimization of Engine Model Parameters Gain and Time Constant for the Cessna Citation $\mathrm{X}$ Business Aircraft Engine
}

\author{
Ruxandra Mihaela Botez ${ }^{1}$, Julian Anthony ${ }^{1}$ and Clementa Hamel ${ }^{1}$ \\ 1 ETS, University of Quebec, Montreal, Que., Canada, H3C1K3 \\ *e-mail address: ruxandra.botez@etsmtl.ca
}

\begin{abstract}
The Research Aircraft Flight Simulator was designed and manufactured by CAE Inc., which is a very well known internationally Aerospace Company in Aircraft Modeling and Simulation Technologies. This simulator was designed following specific requirements of the Laboratory of Applied Research in Active Controls, Avionics and AeroServoElasticity LARCASE team at ETS in Montreal with the aim to be used for research purposes. The authors are the members of the team. This simulator is equipped with a Flight Dynamics Level D open source code, thus it will be used as a Flight Certified Bench Test with the idea to validate Flight Dynamics Models new codes and methodologies. The Cessna Citation $\mathrm{X}$ is the fastest today business aircraft available on the market. However, its engine model is unavailable. For this reason, the authors used this simulator in order to obtain, validate and optimize the main parameters of the engine model such as the time constant and gain variations with altitudes and Mach numbers.
\end{abstract}

\section{Introduction}

The main objective of this research was to determine the variation of both Gain and Time Constant parameters for the RollsRoyce AE3007, which is the Cessna Citation $\mathrm{X}$ engine, in terms of Mach numbers and altitudes. These parameters included the Gain and Time constant of the engine. The Cessna Citation X business aircraft flight dynamics model was identified and validated as explained in [1, 2]. The flight cases are explained in detail in these references. Various optimal control methodologies have been studied for the Cessna Citation $\mathrm{X}$ in $[3,4,5]$.

\subsection{Gain Definition}

The ratio between the output 'Net Thrust $T$ ' and the input 'Thrust Lever Angle (TLA)' was modeled using the following first order transfer function, as shown in $[6,7]$ :
$\frac{N \cdot T}{T L A}=\frac{G}{\tau S+1}$

where the time taken for the $\mathrm{T}$ to drop to $63.2 \%$ of its initial value is defining the time constant $\tau$ in the previous equation (1).

The gain is defined as the $T$ variation resulting from a change in the inlet pressure. The effects of Mach numbers and altitudes on the gain values are described in the following subsections.

\section{Effect of Mach number on the gain}

The Net Thrust can be found by the following equation:

$$
N . T=\left[\dot{m} V_{j}+A_{j}\left(p_{j}-p_{a t m}\right)\right]-\dot{m} V_{i}
$$

from which:

$$
N . T=\dot{m}\left(V_{j}-V_{i}\right)+A_{j}\left(p_{j}-p_{a t m}\right)
$$

Where $\dot{m}$ is the mass flow rate, $V_{j}$ is the exit speed, $V_{i}$ is the inlet or aircraft speed, $A_{j}$ is 
the propelling nozzle area, $p_{j}$ is the static pressure across the propelling nozzle and $p_{i}$ is the atmospheric pressure.

As shown in eq. (3), N.T depends on the difference between the exit and inlet air speeds $V_{j}$ and $V_{i}$. As the aircraft speed $V_{i}$ increases, is evident that the speed difference $\left(V_{j}-V_{i}\right)$ decreases.

Thus, $N$. $T$ decreases, as seen in eq. (3), with the increase in aircraft speed $V_{i}$, and therefore Mach number $M$.

\section{Effect of altitude on the gain}

It is known that the air pressure, density and temperature decreases with an increase in altitude. The gain decreases due to the decrease in mass flow as the air pressure and density decrease. The mass airflow increases with the decrease in the air temperature. At troposphere altitudes above $36.000 \mathrm{ft}$, the net thrust N.T is dependent only on the pressure as temperature remains constant.

\section{Time constant}

\section{Effect of Mach number on the time constant}

The time for the N.T to drop, and therefore the time constant decreases with the increase in Mach number.

\section{Effect of altitude on the time constant}

Depending on the altitudes values, there are low and high altitudes considered. At low altitudes, an increase in altitude causes a decrease in ambient air pressure and temperature resulting in the thrust reduction. At high altitudes, the drop in N.T decreases as the temperature drop decreases.

\subsection{Methods used in the code}

Various engine tests were done, modeled and analyzed. The initial estimation of the parameters was performed in the frequency domain. For example, the Tfest function was used to estimate a continuous time transfer function in the frequency domain. Thus, the gain and time constant were estimated for given input and output data, initial conditions and sampling period.

The Fminsearch function was used as an optimization algorithm in which the cost function was minimized. This cost function estimates the sum of squares errors between all the experimental and modeled $N . T$. values.

\subsection{Validation Technique}

Two validation points were selected in order to have a cloud of points around them; these validation points have been manually chosen from the identification points.

The gain and time constant of these validation points were further interpolated from the graphs of identification points of the Gain versus Mach number and Altitude and from the graph of the Time constant versus Mach number and Altitude. These values were then introduced into the N.T.

The chosen validation points are shown as crosses on the plots below.

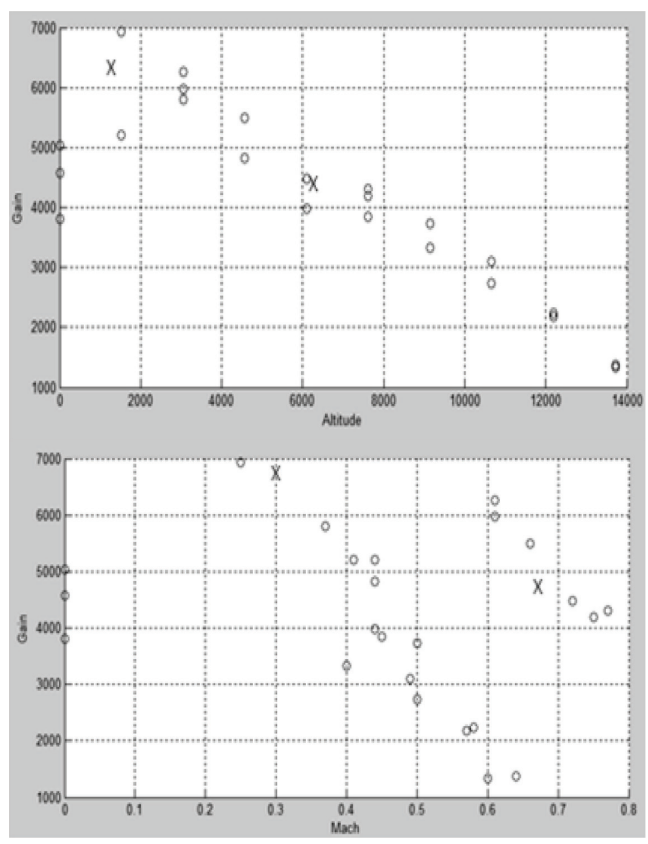

Figure 1. Validation points on the Gain versus Mach number and altitude graphs 


\section{Results}

The tfest function had to be adjusted in order to produce very good estimates of the first order transfer function. Its adjustment was achieved by specifying certain initial conditions and limits to confine the function's estimates. The gradient descent search method was used for the estimation, which is a first order optimization algorithm that finds a function's local minimum by taking steps proportional to the negative of the gradient.

The maximum error was calculated for each test with the aim to analyze the estimation versus the optimization results.

The two following graphs show the variation of time constant and gain with Mach number and altitude before optimization.

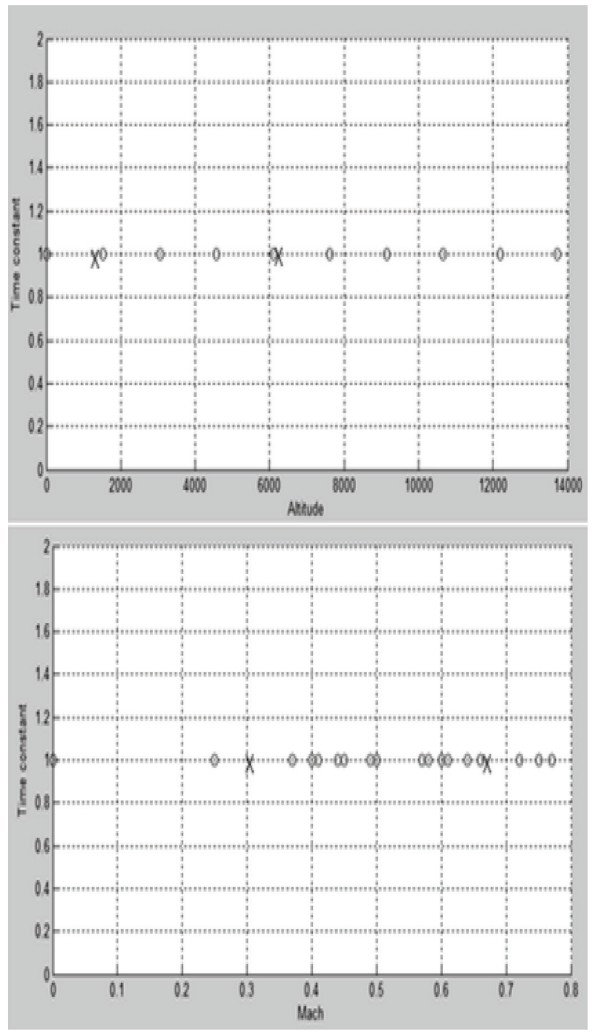

Figure 2. Validation points on the Time Constant versus Mach number and altitude graphs
The time constant had no variation with Mach number and altitude. All engine tests produced a time constant of $1 \mathrm{~s}$ through the estimation algorithm. Even with various alterations to the tfest function, the time constant did not change. The gain on the other hand produced an inverse proportional relationship with the altitude.

As expected, the obtained results were much better after optimization. Less absolute errors were obtained.

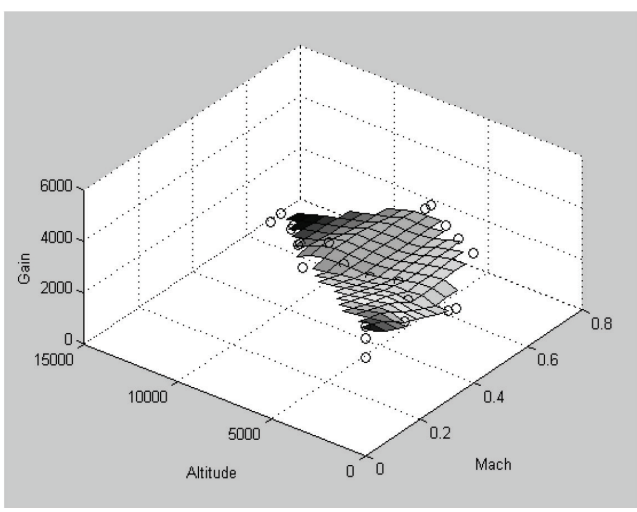

Figure 3. Variation of the gain with the altitude and Mach number before optimization

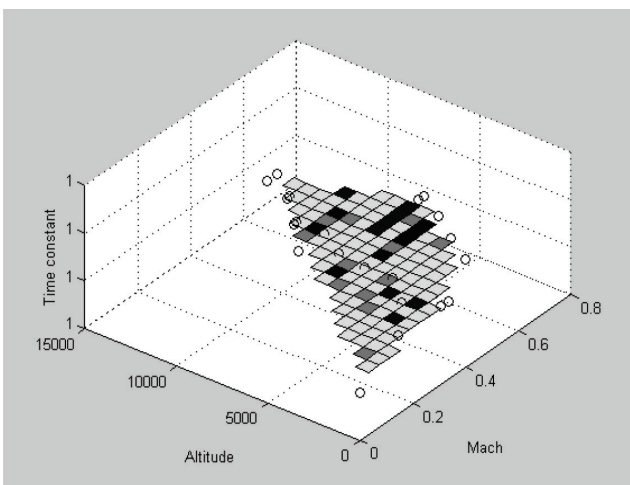

Figure 4. Variation of the time constant with the altitude and Mach number before optimization

The following Figures 5 and 6 show the comparison between the experimental and simulated Net Thrust N.T. variation with time for two tests. The experimental flight 
test data are represented with continuous lines, while the simulated results were represented with lines with discontinuous lines.

\subsection{Identification tests}

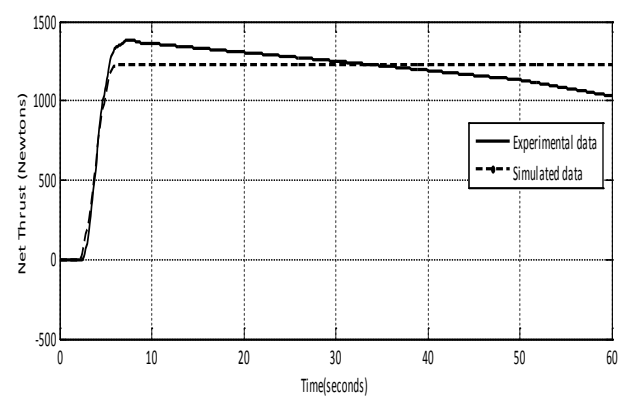

Figure 5. Test 1 Identification (Net Thrust variation with time) with maximum absolute error $=197.68 \mathrm{~N}$

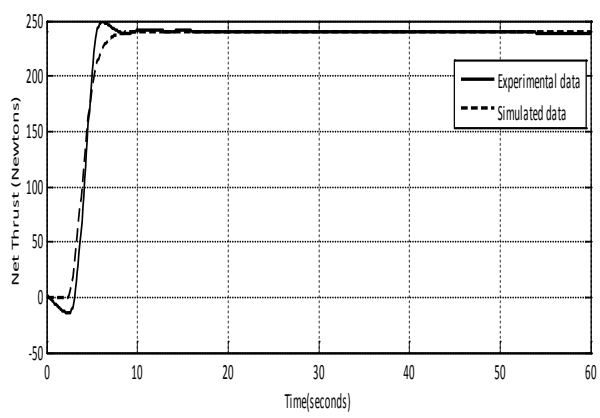

Figure 6. Test 2 Identification (Net Thrust variation with time) with maximum absolute error $=35.82 \mathrm{~N}$

\subsection{Validation tests}

Following the validation of these two tests, two sets of results are presented in next Figures 7 and 8.

The two identification tests are the ones with the highest and lowest maximum initial errors. The highest case showed a $58.98 \%$ decrease in error, while the lowest case showed only a $3.02 \%$ decrease in error.

The reason for the low decrease in error of the worst case can be attributed to the fact that before optimization, the resulting error was already low, therefore the optimization algorithm did not have a major impact.

The percentage decrease in error for the validation tests was considerable as result of the optimization algorithm.

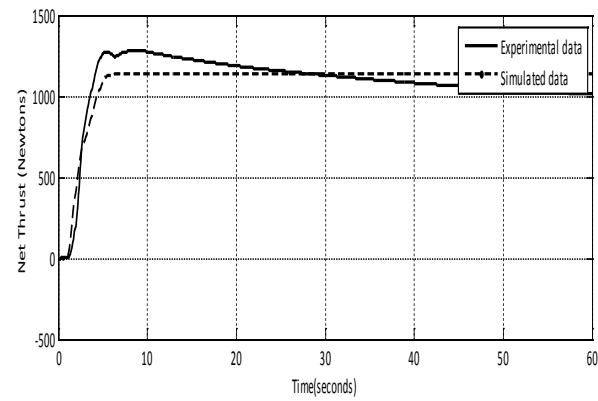

Figure 7. Test 1 Validation (Net Thrust variation with time) with maximum absolute error $=210.37 \mathrm{~N}$

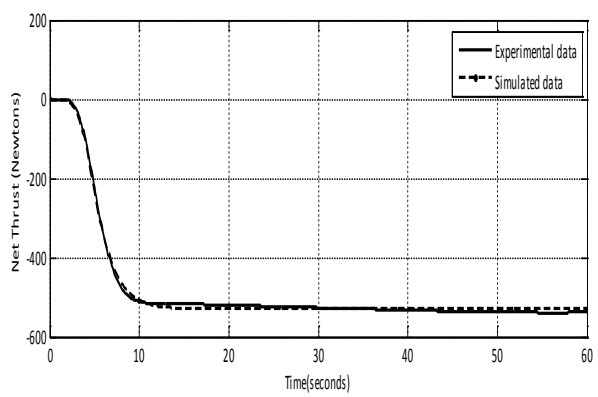

Figure 8. Test 2 Validation (Net Thrust variation with time) with maximum absolute error $=16.32$ $\mathrm{N}$

Since good validation results are essential for a successful model, this optimization algorithm was proven to be very important. The variations of the time constant and the gain with altitude and Mach numbers were improved after optimization as seen in following Figures 9 and 10.

The new optimized gain was almost unaltered as compared to its initial nonoptimized value. Similar relationships of the new optimized gain with Mach number and altitude can be observed, while the new optimized time constant does not vary with Mach number. The new optimized time 
constant decreases with altitude for altitudes higher than $6,000 \mathrm{~m}$.

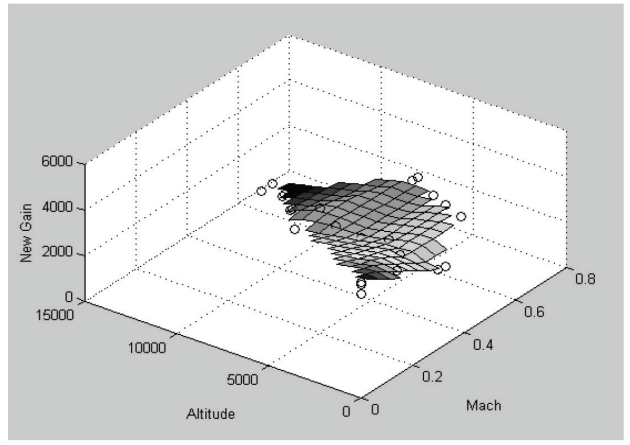

Figure 9. Gain variation with altitude and time improvement after optimization

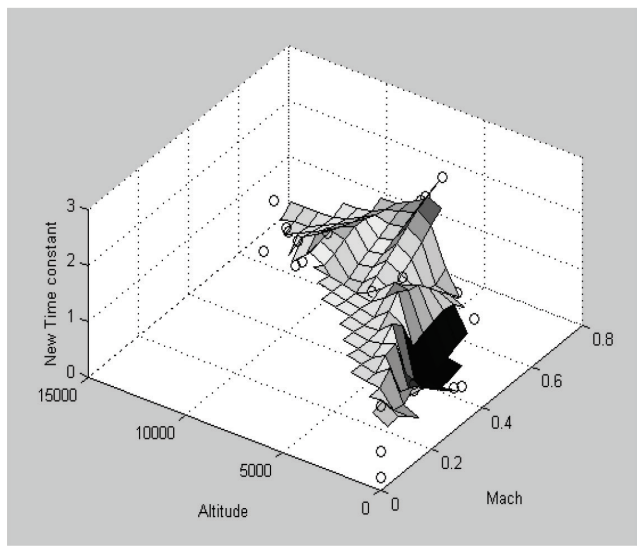

Figure 10. Time constant variation with altitude and time improvement after optimization

\subsection{Analysis of errors}

In Figure 11, it can be seen that the error after optimization was much smaller than the error before optimization, thus our new optimization method gave good results.

Hence, the first order transfer function model was suitable for the Rolls-Royce AE3007 engine. The estimation and optimization combined algorithms have accomplished the objectives of this project, and gave excellent results.
Other identification methods for the engine or aircraft are described in $[8,9,10,11,12$, $13,14,15]$.

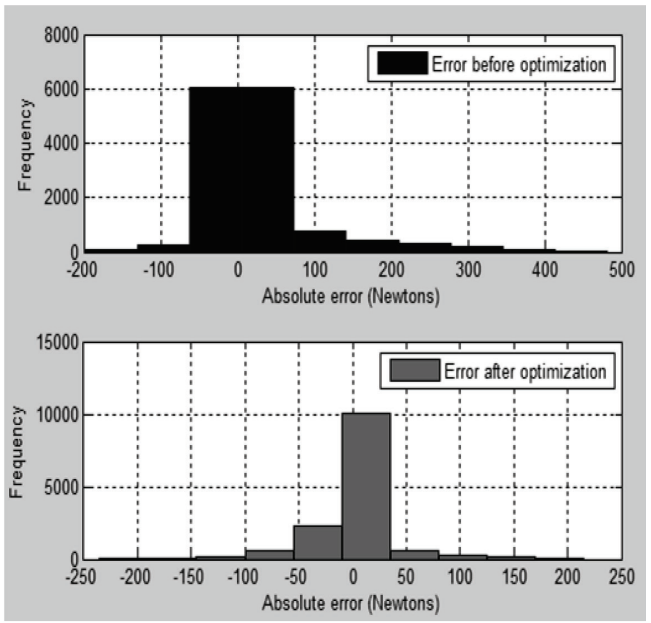

Figure 11. Gain variation with altitude and time improvement after optimization

\section{Acknowledgment}

The authors would like to thank to the NSERC for the funding received for this research. Thanks are dues to the CAE Inc. team led by Mr Ken Dustin for their support related to the Aircraft Research Flight Simulator, as well as to Mrs Odette Lacasse and $\mathrm{Mr}$ Oscar Carranza for their support and motivation.

\section{References}

1) Hamel, C., Sassi, A., Botez, R. M. and Dartigues, C., Cessna Citation X Aircraft Global Model Identification from Flight Tests, SAE International Journal of Aerospace, Vol. 6(1), (2013), 106.

2) Hamel, C., Botez, R. M. and Ruby, M., Cessna Citation X Airplane Grey-Box Model Identification without Preliminary Data, Proceedings of the SAE 2014 Aerospace Systems and Technology Conference, Cincinnati, United States, September 24-26, (2014).

3) Boughari, Y., Botez, R. M., Ghazi, G. and Theel, F., Evolutionary Algorithms for Robust Cessna Citation X Flight Control, SAE 2014 Aerospace Systems and 
Technology Conference, Cincinnati, United States, September 24-26, (2014).

4) Boughari, Y., Botez, R. M., Theel, F. and Ghazi, G., Optimal Flight Control on Cessna X Aircraft using Differential Evolution. International Association of Science and Technology for Development IASTED Modelling, Identification and Control (MIC 2014), Innsbruck, Austria, February 17-19, (2014).

5) Ghazi, G. and Botez, R. M., New Robust Control Analysis Methodology for Lynx Helicopter and Cessna Citation X Aircraft Using Guardian Maps, Genetic Algorithms and LQR Theories Combinations, American Helicopter Society 70th Annual Forum \& Technology Display, Montreal, Canada, May 20-25, (2014).

6) Rodriguez Fajardo, L. and Botez, R. M., Generic new modelling technique for turbofan engines thrust, The AIAA Journal of Propulsion and Power, Vol. 29(6), pp. 1492-1495.

7) Rodriguez Fajardo, L., Botez, R. M., Civil turbofan engines semi-empirical thrust generic model, IECON 2012 Conférence, Montréal, Québec, Canada, 25-28 October, (2012).

8) De Jesus Mota, S.., Nadeau Beaulieu M. and Botez, R. M., 2009, Identification of a MIMO State Space Model of an F/A-18 Aircraft using a Subspace Method. The Aeronautical Journal, Vol. 112(1142), (2009), 183.
9) Kouba, G., Botez, R. M. and Boëly, N., Fuzzy Logic Method used in the F/A-18 Aircraft Model Identification, Journal of Aircraft, Vol. 47(1), (2010), 10.

10) Boëly, N., Botez, R. M. and Kouba, G., Identification of a Nonlinear F/A-18 Model by use of Fuzzy Logic and Neural Network Methods. Proceedings of the Institution of Mechanical Engineering, Part G: Journal of Aerospace Engineering, Vol. 225, (2010), 559.

11) Jategaonkar, R. V., Flight vehicle system identification a time domain methodology. Reston, VA: American Institute of Aeronautics and Astronautics, (2006).

12) Klein, V., Aircraft system identification theory and practice. Reston, VA: American Institute of Aeronautics and Astronautics, (2006).

13) Tischler, M. B., Aircraft and rotorcraft system identification engineering methods with flight-test examples. Reston, VA: American Institute of Aeronautics and Astronautics, (2006).

14) Morelli E. A. and Klein, V., 2005, Application of system identification to aircraft at NASA Langley Research Center, Journal of Aircraft, Vol. 4(1), (2005), 12.

15) Jategaonkar, R. V., Fischenberg, D. and Gruenhagen, W. V., 2004, Aerodynamic modeling and system identification from flight data - Recent applications at DLR, Journal of Aircraft, Vol. 41(4), (2004). 